\title{
Heat Preconditioning Protects Skin Pressure Injury and Reduces Incidence of Intraoperatively Acquired Pressure Ulcers in Both Humans and Animals
}

\section{Huiping Xue}

Nagano College of Nursing

\section{Shuwen Chang}

Nagano College of Nursing

Ping Hou

Nagano College of Nursing

Ping Xie

Northern Jiang Su people's Hospital

Yajie Chen

University of Yamanashi

\section{Yanwei Wang}

Nagano College of Nursing

\section{Daiji Miura}

Nagano College of Nursing

Jianglin Fan

University of Yamanashi

Jingyan Liang

Yangzhou University Medical College

Akio Kitayama

Nagano College of Nursing

\section{Fang Fang}

Northern Jiang Su people's Hospital

Haijuan Yuan

Northern Jiang Su people's Hospital

Xiaoling Wu

Northern Jiang Su people's Hospital

\section{Xiaolin Zhang}

Northern Jiang Su people's Hospital

Jing Wang

Northern Jiang Su people's Hospital

Ning Ding

Northern Jiang Su people's Hospital 


\section{Can Zhang}

Northern Jiang Su people's Hospital

\section{Xiuyun Sun}

Northern Jiang Su people's Hospital

En Takashi ( $\sim$ takashi@nagano-nurs.ac.jp )

Nagano College of Nursing

\section{Research Article}

Keywords: Heat Preconditioning, Intraoperatively Acquired, Humans and Animals

Posted Date: April 23rd, 2021

DOI: https://doi.org/10.21203/rs.3.rs-380147/v1

License: (c) (i) This work is licensed under a Creative Commons Attribution 4.0 International License. Read Full License 


\section{Abstract}

To examine the preventive effect of heat preconditioning (HPC) on intraoperatively acquired pressure ulcers (IAPU) in surgical patients and explore the mechanisms of HPC on pressure injury (PI) in rat skin. We generated a skin PI model using hairless rats and treated these rats with HPC. Skin injury size was measured and the expression of heat shock proteins (HSPs) in the skin were examined by real-time RT PCR, Western blotting, and immunohistochemical staining. For human studies, 118 surgical patients were randomly divided into HPC and control groups. We measured sacral skin temperature, sacral pressure and compared the PI prevalence rate. HPC treatment significantly reduced both area $(p=0.007, p=0.007, p$ $=0.012)$ and incidence $(p=0.025, p=0.019, p=0.012)$ of $\mathrm{PI}$ in rats on the second, third and fourth days. HSP27 expression was increased in HPC group. Immunohistochemical staining showed that HSP27 was distributed in all types of dermal cells with increase in basal cells. In human studies, HPC treatment increased sacral temperature $(p<0.001)$. The incidence of IAPUs was significantly decreased by $75 \%$ in HPC group. HPC can reduce the incidence of PI in both experimental animals and humans. Beneficial effects of HPC were possibly mediated by upregulation of HSP27.

\section{Introduction}

Pressure injury (PI) refers to local lesions of the skin and deep soft tissue caused by pressure ${ }^{1}$ and is a global concern for surgery patients. ${ }^{2} \mathrm{PI}$ not only affects the prognosis of patients, but also brings about serious health and economic losses. ${ }^{3}$ The incidence of pressure ulcers has decreased owing to the implementation of preventive measures, and the prevalence of $\mathrm{PI}$ is currently $3.38 \%$ in hospitals. ${ }^{4}$ The risk of PI during surgery is particularly high because patients stay in the same position for a long time. One study showed that the incidence of intraoperative acquired pressure ulcers (IAPUs) was as high as 4.7$66 \%{ }^{5}$

IAPUs refer to skin damage caused by pressure that occurs during surgery. ${ }^{6}$ While factors such as the requirement for special positions during surgery ${ }^{7}$ and prolonged operation time are associated with IAPUs ${ }^{8}$, its incidence remains high. ${ }^{5}$ Therefore, it is urgent to identify novel methods for preventing IAPUs.

Ischemic preconditioning (IPC) was first proposed by Merry et al. to reduce the fatal injury rate associated with myocardial ischemia. ${ }^{9}$ Heat preconditioning (HPC) is derived from IPC, and can stimulate the heat shock response by repeatedly heating a local area. HPC has been proposed to prevent various stressinduced changes in cells and tissues, including oxidative stress, and it can protect organs such as intestines and lungs from oxidative stress. ${ }^{10}$ Recently, several studies showed that HPC reduces necrosis of the surrounding skin tissue after breast reconstruction. ${ }^{11,12}$ The molecular mechanism for these effects is thought to be related to heat shock proteins (HSPs), ${ }^{11}$ although this hypothesis has not yet been fully investigated. Among the many HSPs, HSP27 appears to participate in the anti-apoptosis response and protects the heart and kidney from ischemia/reperfusion injury. ${ }^{13,14}$ HSP27 is widely distributed in the skin ${ }^{15}$ and is considered to be a sensitive marker for skin irritation; ${ }^{16}$ it is continuously expressed in epidermal cells of the normal skin. ${ }^{17}$ We therefore hypothesized that HSP27 may participate in protection 
against IAPU. To test this hypothesis, we generated a PI model using hairless rats. The current study was performed to examine whether HPC exerts a protective effect in both humans and animal models.

\section{Results}

\section{Increased skin temperature after HPC in rats}

The skin temperature in the HPC group was significantly higher than that of the control group (Fig. 1), suggesting that heat pretreatment increased skin temperature. The skin temperature of the HPC group was $38.88 \pm 1.90^{\circ} \mathrm{C}$ throughout the experiments, ranging from $37.13^{\circ} \mathrm{C}$ to $40.91^{\circ} \mathrm{C}$. At the same time, we found that the temperature of the heat transfer sheet was $40.7 \pm 0.6^{\circ} \mathrm{C}$; thus, there was a temperature difference of nearly $2^{\circ} \mathrm{C}$ between the heat transfer sheet and the skin temperature of the HPC group. However, the body seemed to retain heat after each treatment, and the temperature difference between the skin and the heat transfer sheet gradually decreased to $1^{\circ} \mathrm{C}$ during the second and third treatment cycles. Therefore, the temperature of human skin treated with heat is expected to rise to $40.5^{\circ} \mathrm{C}$ or higher. The rectal temperature was $36.01 \pm 0.78^{\circ} \mathrm{C}$, ranging from $33.81^{\circ} \mathrm{C}$ to $35.93^{\circ} \mathrm{C}$. The skin temperature of the control group was 34.90 $\pm 0.57^{\circ} \mathrm{C}$, ranging from $34.33^{\circ} \mathrm{C}$ to $37.08^{\circ} \mathrm{C}$.

\section{Area and percentage of PI affected by HPC}

HPC after 1, 2, 3, and 4 days enhanced the healing process, as shown by the gross appearance of pressure injury compared with the control group. The incidence of PI in the HPC group was lower than in the control group, on the second, third and fourth days, the incidence of PI in the HPC group was lower than that in the control group, and the differences were statistically significant $(p=0.025, p=0.019, p=0.012)$. And the area of PI in the HPC group was clearly smaller than in the control group, on the second, third and fourth days, the area of PI in the HPC group was smaller than that in the control group, and the differences were statistically significant $(p=0.007, p=0.007, p=0.012)$ (Fig. 2).

\section{Upregulation of HSPs induced by HPC}

As shown in Fig. 3, in normal epidermis HSP-27 was localized in spinous cells and some granulosa cells, and slightly increased after heating. In injured areas, HSP-27 was highly expressed in all layers of cells in the epidermis, including spinous cells, granulosa cells, and basal cells, and the distribution of hair follicle cells was also enhanced. There was no HSP-27 expression in basal cells in the control group after injury. After HPC treatment, we compared HSP expression using real-time RT-PCR and found that among the five HSPs $(10,27,40,70$ and 90), mRNA expression of HSP27 in the HPC group was the most highly upregulated (Fig. 4, top panel). The significant upregulation of HSP27 was also observed at the protein level using western blotting analysis (Fig. 4, bottom panel). To observe the effects of HPC in the skin, we performed immunohistochemical staining for HSP-27.

\section{Improved sacral temperature and reduced incidence of IAPUs in patients after HPC}


A total of 118 patients participated in the current study. There was no difference in age, gender, medical history, body position, hemoglobin or albumin levels, operation duration, or BMI between the groups (Table 1).

HPC treatment significantly improved the sacral temperature $(p<0.001)$, and the average temperature in the HPC group was approximately $2^{\circ} \mathrm{C}$ higher than in the control group (Table 2 ).

In the control group, 8 patients had PIs (stage1: 6 cases; stage2:2 cases) and the incidence was $13.6 \%$. Two cases were healed at 7 days after operation, both of which were stage1, and healed at 2 days after operation. In the HPC group, 2 patients had PIs (stage1 and stage2: each 1 case), and the incidence was $3.4 \%$ ( $p=0.047$ vs control group). One of them was recovered after 1 day of operation (stage 1 ), and the other was healed after 7 days (stage2). Healing rate was higher in the HPC group compared with the control group $(p=0.053)$. Although the difference of healing rate was not statistically significant, this may have been due to the small sample size (Table 3).

The sacral pressure was $38.34 \pm 0.89 \mathrm{mmHg}$ in the HPC group and $36.56 \pm 1.03 \mathrm{mmHg}$ in the control group, a non-significant difference $(p=0.196)$.

\section{Discussion}

The main finding of the present study is that HPC protects skin from PI. For skin tissue that will be subjected to long-term surgical compression, local HPC can reduce the occurrence of PI, and this protective mechanism is achieved by stimulating HSP27 expression in tissues. This was also the first study to use HPC to prevent IAPUs in patients undergoing surgery in the supine position. Local HPC (using $43^{\circ} \mathrm{C}$ warm water in a hot water bag) on the sacral skin can reduce the incidence of IAPUs in elective surgery patients.

At present, measures such as the use of dressings and skin emollients, nutritional supplements, physical support, and repositioning are commonly used to prevent IAPUs. However, there is no high-quality report on the relative effectiveness of these products. ${ }^{18}$

When organisms are exposed to harmful external stimuli (such as high-temperature heat shock, trauma, infection, and hypoxia), they quickly initiate a self-protective response that involves the activation of genes that encode protective HSPs. ${ }^{19}$ HSPs are a group of proteins expressed in the heat shock response. The HSP family can be divided into seven categories based on relative molecular weight: small HSP family, HSP10/60 family, HSP40 family, HSP70 family, HSP90 family, HSP110 family, and CCT family. ${ }^{20}$ There is evidence that HSP27 protects cells from apoptosis induced by various stimuli in vivo and in vitro. The expression of HSP27 can induce the expression of anti-apoptotic factors in the skin, reduce oxidative stress and aging, and thereby alleviate skin damage. ${ }^{21} \mathrm{HSPs}$ play an important role in maintaining protein homeostasis and cell survival. Small-molecular-weight HSPs play a particularly important role, especially HSP27. Muramatsu et al. ${ }^{22}$ measured the expression of HSP27 by immunofluorescence in normal human skin after HPC for 1 hour, and found that HSP27 plays an important role in resistance to various 
environmental stresses in human skin. Small-molecular-weight HSPs can combine with a variety of proteins to enhance cellular protection to a range of stressors. ${ }^{23}$ Our results were consistent with these findings.

Many studies in both humans and animals have applied HPC to the skin, but the efficacy of HPC in preventing PI has not been clearly established. Several studies have confirmed that local heating of the skin can significantly improve tissue survival. ${ }^{24,25}$ The protective effects observed in these studies are thought to be due to the upregulation of HSP70 and HSP32 expression induced by high temperatures. In addition, Mehta et al. ${ }^{11}$ heated the skin of breast cancer patients in skin-sparing mastectomy and found that HPC could reduce skin necrosis and duration of hospital stay. In the present study, we found that the incidence of $\mathrm{PI}$ and the area of $\mathrm{PI}$ could be significantly reduced by heating rat dorsal skin. In human patients, we found that HPC could significantly reduce the incidence of IAPUs.

Ischemia/reperfusion injury is the main cause of $\mathrm{PI}^{26,27}$ There is a great deal of evidence showing that HPC can protect heart, ${ }^{28}$ liver, $_{,}^{29}$ and kidney ${ }^{30}$ tissue from hypoxia and ischemia/reperfusion injury in rats. Overexpression of HSP70 plays a direct role in improving myocardial and renal tolerance to ischemia/reperfusion injury in the heart and kidney. ${ }^{31,32}$ In hepatic ischemia/reperfusion injury, the improvement in microcirculation resulting from HPC-induced HSP70 expression is the main mechanism for these effects. ${ }^{29}$ However, other studies found that HSP70 was not detected during intestinal heat preconditioning, ${ }^{33}$ or in whole body hyperthermia at $40^{\circ} \mathrm{C} .{ }^{34} \mathrm{HSP} 72, \mathrm{HSP} 60$, and HSP27 expression has been found to increase after HPC treatment of normal human skin, but the expression of HSP9O and HSP110 did not increase. ${ }^{35}$ However, some studies have also suggested that in chronic ischemic skin, HPC reduces ischemic tissue necrosis by improving microcirculation, an effect mediated by HSP32. ${ }^{36}$ Therefore, the mechanism underlying the protective effects of HPC remains controversial.

To elucidate the mechanism underlying the protective effects of HPC on Pl, including the heat shock proteins involved, we observed both groups for 4 days after compression and selected tissues for histological analysis after 1 day. We chose the 1 day timepoint based on our observation that whether PI occurs tends to be determined in both groups after one day. The results of this study suggest that the protective effects of HPC on ischemic skin are related to the induction of HSP27, which is consistent with the results of Boxman et al. ${ }^{37}$ Boxman et al. ${ }^{40}$ used chemicals to stimulate human skin and identified seven proteins as potential new epidermal markers for skin irritation. Among these seven proteins, HSP27 was identified as the most significantly upregulated, suggesting that HSP27 can be used as a sensitive marker for skin irritation. However, there were some differences between the present results and the HSP70 expression induced by HPC in the study mentioned above. In this previous study, the temperature used for $\mathrm{HPC}$ was $43^{\circ} \mathrm{C}$. However, in the current study, the final temperature of the heat transfer sheet material was $40.7 \pm 0.3^{\circ} \mathrm{C}$ owing to the use of three 30 minutes cycles at room temperature. While the temperature used in the present study was lower than those used in previous studies, it is also capable of inducing HSP. Our study has the following limitations: In the whole process of animal experiment, the sample size may be small. In addition, due to the limitation of the research conditions, we failed to analyze the microcirculation including arterioles, venules and capillaries in the compressed parts of the rats in the control group and the intervention group. During the human studies, there were fewer patients with Intraoperatively Acquired 
Pressure Ulcers, which may be related to the cotton cushion on the operating table to reduce the compression of sacrococcygeal. Considering the follow-up and survival rate, some patients with higher risk operations such as cardiac surgery and craniocerebral surgery were not included in the study. The main group of this study was digestive tract related diseases (esophageal cancer, gastric cancer, intestine), pancreatic disease, biliary disease, etc.). The sample size is not representative of all kinds of inpatients. In the future, we can consider increasing the sample size, and further explore the mechanism that HPC can alleviate the occurrence of PI.

In this study, we found that HPC treatment can significantly improve body temperature and enhance the skin healing process in both a rat PI model and surgical patients, indicating that HPC has protective effects against PI. This effect appears to be mediated by HSP27 upregulation. HPC can be implemented preoperatively in surgical patients and does not require intraoperative change of body position. Therefore, HPC may be a novel and effective means of preventing IAPUs for elective surgery patients.

\section{Methods}

Animal experiments were conducted in accordance with Japanese laboratory animal management policy and animal protection law, and were approved by the Committee for the Ethics of Animal Experiments of the Nagano College of Nursing (No. 2018-3). Human studies were designed and conducted in accordance with the principles of the Declaration of Helsinki and approved by the Ethics Committee of Northern Jiang Su People's Hospital in 2018 (ethics approval number: 2018068). The registration number for human studies was ChiCTR2000041301 (23 / 12 /

2020;http://www.medresman.org.cn/pub/cn/proj/projectshow.aspx?proj=7389). Informed consent was obtained from all patients. The study was carried out in compliance with the ARRIVE guidelines. All methods were performed in accordance with relevant guidelines and regulations.

\section{Animal studies}

A total of 15 rats (HWY/Slc; 10-week-old; 230-250 g body weight; SLC Inc., Shizuoka, Japan) were included in the study, and there were no accidental deaths. The PI method was modified from Stadler as previously described. ${ }^{38}$ Seven rats were used for histological examinations and HSPs expression analyses, and eight were used to observe the progression of Pl. Each rat was placed in a single cage at an ambient temperature of $23^{\circ} \mathrm{C}$ and a humidity of $35 \%$ with a 12 -h light/dark cycle. During this process, each rat was given adequate water and nutrition.

\section{Heat Preconditioning}

Heat preconditioning was on the right dorsal skin as HPC group and the left was the control group. The HPC group was heated by hot plate, equipped with heat transfer sheet and covered by insulated board with a $3 \mathrm{~cm} \times 5 \mathrm{~cm}$ rectangle hole in the middle. The hot plate temperature was set to ensure the sheet material does not exceed $43^{\circ} \mathrm{C}$, which provides heat application to a supraphysiological level without causing a burn. Heat shock response induced by hyperthermia $\leq 43^{\circ} \mathrm{C}$ has been used in many studies, which can inhibit cell apoptosis, improve cell survival rate, reduce DNA strand breakage, and avoid the damage caused by 
high temperature. ${ }^{39}$ Moreover, in this study, the temperature of the heat transfer sheet was always lower than $42{ }^{\circ} \mathrm{C}$. The temperature sensor monitors the temperature of the sheet material in real time, and automatically collects the temperature of the sheet material every $30 \mathrm{~s}$, so that it does not exceed $43^{\circ} \mathrm{C}$. The rats were sedation with a mixture of medetomidine hydrochloricde $(0.15 \mathrm{mg} / \mathrm{kg}$ Domitor, Zenoaq, Fukushima, Japan), midazolam hydrochlride (2.0 mg/kg; Dormicaum; Astellas Pharma, Tokyo, Japan) and butorphanol ( $2.5 \mathrm{mg} / \mathrm{kg}$; Vetorphale; Meiji Seika Pharma Co., Ltd. Tokyo, Japan) by intravenous injection during the process of heating. The right dorsal was then heated up for $3 \times 30$ minutes, interrupted by cycles of 30 minutes each at room temperature $\left(23^{\circ} \mathrm{C}\right)$ during which the rats were taken off the heating sheet. The left dorsal was Non-heated. At the same time, the temperature of skin, rectum and heat transfer sheet of the rats was monitored and recorded with a temperature sensor (LT-8A, Gram Corporation, Saitama, Japan) during the heating period. The HPC was pretreated 1 day in advance before the pressure injury (PI) model was prepared.

\section{Preparation of PI Model}

Rats were anesthetized intravenously with a mixture of medetomidine hydrochloricde $(0.15 \mathrm{mg} / \mathrm{kg}$ Domitor, Zenoaq, Fukushima, Japan), midazolam hydrochlride (2.0 mg/kg; Dormicaum; Astellas Pharma, Tokyo, Japan) and butorphanol (2.5 mg/kg; Vetorphale; Meiji Seika Pharma Co., Ltd. Tokyo, Japan) during PI modeling. PI was established by controlling the time of pressure action using magnet oppression skin to simulate ischemia-reperfusion injury. After anesthesia, the skin of symmetrical part of the middle line of the back was lifted gently, and the skin was oppressed with a neodymium magnet (NE002, Niroku Seisakusho Co., Ltd, Japan) of $12 \mathrm{~mm}$ in diameter and $3 \mathrm{~mm}$ in thickness (Adsorption force $=2 \mathrm{~kg} \times 2$ ) for 4.5 hours.

After 4.5h of oppression, pressure on rats was relieved immediately and lesions in the dorsal skin in both groups were photographed by a digital camera (Optio, WG-III, PENTAX) for macroscopic observation at 0,1 , 2, 3, and 4 days (Supplementary Figure S1). The whole experiment lasted for four days. During this period, the state of rats was evaluated every 2 hours on the first day, every 4 hours on the second day, and every 6 hours on the third and fourth days. Finally, computer-aided image analysis (ImageJ software, National Institutes of Health) was used to circle the area where the lesion of skin in the images, and the corresponding area of damage was calculated $\left(\mathrm{cm}^{2}\right)$ to quantitatively analyze the effect of HPC on PI. At the end of the experiment, rats were euthanized by excessive injection of pentobarbital $(120 \mathrm{mg} / \mathrm{kg}, \mathrm{IP})$.

\section{Histological observation and immunohistochemical staining}

At the one day after the experiment, the rats were sacrificed by injection of an overdose of sodium pentobarbital solution. For histological examination, the skin specimens were fixed in $10 \%$ buffered formalin, embedded in paraffin, and sections ( $3 \mu \mathrm{m}$ thick) were stained with hematoxylin and eosin (H\&E). Immunohistochemical staining was performed using an anti-HSP27 antibody (dilution 1:100, sc-13132, Santa Cruz Biotechnology Inc.) as previously described. ${ }^{40}$ The $3 \mu \mathrm{m}$-thick paraffin sections were first deparaffinized and rehydrated, then incubated in $0.3 \% \mathrm{H}_{2} \mathrm{O}_{2}$ in ethanol for $30 \mathrm{~min}$ at room temperature to block endogenous peroxidase activity. Antigen retrieval was performed as follows: all slides were incubated in retrieval solution $(1.9 \mathrm{ml}$ of $0.1 \mathrm{M}$ citric acid solution $+8.3 \mathrm{ml}$ of $0.1 \mathrm{M}$ sodium citrate hydrate solution + $90 \mathrm{ml} \mathrm{H}_{2} \mathrm{O}$ ) and autoclaved at $125^{\circ} \mathrm{C}$ for $5 \mathrm{~min}$. The primary antibody was incubated at $4^{\circ} \mathrm{C}$ in a moist box 
overnight, and the signal was visualized with 3-amino-9-ethylcarbazole. Hematoxylin was used for nuclear staining and slides were observed by light microscopy (BY 41TF Olympus Optical Co., Tokyo, Japan). Two lesions were selected for histological observation: the PI area (injury area) and a non-injury area ( $5 \mathrm{~mm}$ away from the PI border). Mouse non-specific IgG was as the negative control to confirm the specific of these antibodies (data not show).

\section{RT-PCR analysis}

To analyze HSP gene expression, we performed quantitative real-time PCR. Total RNA was extracted from wounded skin using TRIzol solution (Invitrogen, Carlsbad, CA, USA), and CDNA was synthesized with a reverse transcription kit (iScript cDNA Synthesis kit, Bio-Rad, Richmond, CA, USA). The relative expression levels of HSP-10, HSP-27, HSP-70, and HSP-90, along with GAPDH, were analyzed by real-time PCR using the SsoFast EvaGreen system (Bio-Rad). The sequences of the primers used were as follows: HSP-27, 5'AAAGCAGT CACACAATCAGCG G-3' (forward) and 5'-TACTTGGCTCCAGACTGTTCCG-3' (reverse); HSP-70, 5'GTGCTGCGGATCATCAACGAG-3' (forward) and 5'-AACTCCT CCACGAAGTG GCTC-3' (reverse); HSP-90, 5'CCTGGTGGTGCTGCTGTTTG-3' (forward) and 5'-ATGCGGTAGATGCGGTTGGA-3' (reverse); and GAPDH, 5'CCCTGGCCAAGGTC ATCCAT-3' (forward) and 5'-TCACGCCACAGCTTTCCAGA-3' (reverse).

\section{Western blotting}

For western blotting analysis, wounded skin was homogenized in ice-cold RIPA buffer. Equal amounts of crude protein $(30 \mu \mathrm{g})$ were fractionated by electrophoresis on 10\% SDS-polyacrylamide gels under reducing and denaturing conditions. Next, proteins were transferred to $0.2 \mu \mathrm{m}$ pore-size nitrocellulose membranes (Bio-Rad), and these membranes were incubated with HSP27antibodies (1:1000 dilution, sc-13132, Santa Cruz Biotechnology Inc., Santa Cruz, CA, USA) or $\beta$-actin (1:2000, sc-81178, Santa Cruz Biotechnology Inc.) at $4^{\circ} \mathrm{C}$ overnight, then washed three times with PBST ( $0.1 \%$ Tween 20 in $1 \mathrm{X}$ phosphate-buffered saline) and reacted with horseradish peroxidase-conjugated secondary antibodies followed by enhanced chemiluminescence detection. The band density was quantified with ImageJ software.

\section{Human studies}

The human study (randomized controlled trial) procedure is shown in Supplementary Figure S2. A total of 118 patients who underwent elective surgery at Northern Jiang Su people's Hospital were consecutively enrolled from January 2019 to December 2019. All participants were intubated and administered a standardized anesthesia protocol, including general anesthesia with total intravenous or inhalation anesthesia using sevoflurane. Inclusion criteria were supine position, 18 years old or older, elective surgery under general anesthesia, and expected surgical duration more than $2 \mathrm{~h}$. Exclusion criteria were patients with preoperative pressure injuries, peripheral neuropathy, paralysis, muscular diseases, dermatosis, or expected hospital stay $<3$ days. All participants were randomly divided into the HPC and control groups according to their day of admission by authors. The following instruments were used for clinical investigations: pressure measuring instrument (Palm Q, Cape Co., Yokosuka, Japan), temperature measuring instrument (infrared thermometer AD-5611A, A\&D Company, Tokyo, Japan), hot water bag (volume, $2000 \mathrm{ml}$; length, $31 \mathrm{~cm}$; width, $20 \mathrm{~cm}$; high-density PVC material, Beijing, China), and water temperature meter $\left(0-50^{\circ} \mathrm{C}\right.$ precision thermometer, Xinglong Instrument Factory, Beijing, China). 


\section{Procedures}

The HPC group received heat preconditioning (HPC) for $16 \mathrm{~h}$ (starting from $4 \mathrm{pm}$ ) before surgery. There was no intervention in the control group. The HPC was administered using a hot water bag filled with $43^{\circ} \mathrm{C}$ warm water, which was applied to the sacrum for three 30-min periods separated by 30-min intervals. We measured the skin temperature of the sacral site before and after HPC. The skin temperature of the sacrum in the control group was measured during the same time periods as the HPC group.

\section{Intraoperative phase}

When patients entered the operating rooms, the sacral pressure was measured before general anesthesia. Average values from three measurements at each site were recorded.

\section{Postoperative phase}

After surgery, the patients were transferred to wards. The researchers followed up with patients from the time after surgery to 7 days. Pls were recorded during the observation period.

PI staging was determined according to the NPIAP classification. ${ }^{1}$

\section{Statistical analysis}

SPSS 22.0 statistical software was used to analyze all data. The continuous data were expressed as mean and standard deviation (SD), numerical values. The categorical data were expressed as percentages, frequencies, or proportions. T-tests and chi-square tests were performed to compare results between the HPC group and control group. $P<0.05$ was used as the threshold for statistical significance. Tests were two-tailed.

\section{Declarations}

\section{Acknowledgments}

The work was supported by grants-in-aid for scientific research from the Ministry of Education, Culture, Sports and Technology, Japan, 18K10510, and project of Jiangsu provincial Six Talent Peaks, China, WSN082. The work was also supported by project of Nantong Science and technology project, China, MSZ0030.

The authors thank Chen Hongmei, Mao Xine (College of Nursing, Yangzhou University, China), Jing jing (Changzhou Health Higher Vocational-Techniacal School, China) , all the operating room nurses and Mrs Wenfang Deng in Trauma department of orthopedics (Northern Jiang Su people's Hospital, China) for invaluable assistance.

\section{Author contributions}

Conceived and designed the experiments: H.P.X., S.W.C., P.H., P.X., E.T., J.Y.L.

Performed the animal experiments: H.P.X., P.H., D.J.M., Y.W.W., Y.J.C., E.T. 
Performed the human experiments: S.W.C., P.X., F.F., H.J.Y., C.Z., X.L.W., X.L.Z., J.W., N.D., X.Y.S., E.T.

Analyzed the data: H.P.X., S.W.C., P.H., D.J.M., Y.J.C., Y.W.W., J.L.F., J.Y.L., A.K., E.T.

Contributed reagents/materials/analysis tools: H.P.X., P.H., Y.J.C., Y.W.W., J.L.F., J.Y.L., A.K., E.T.

Wrote and revised the paper: H.P.X., S.W.C., P.H., Y.J.C., J.L.F., J.Y.L., A.K., E.T.

\section{Competing interests}

The authors report no conflicts of interest.

\section{References}

1. NPIAP. Pressure injury stages. 2016 Apr 8 [cited 31 March 2021]. In: NPIAP [Internet]. Available at: http://www.npuap.org/resources/educational-and-clinical-resources/npuap-pressure-injury-stages/ .

2. Spruce L. Back to basics: preventing perioperative pressure injuries. Aorn J. 105,92-99(2017).

3. Schuurman, J.P. et al. Economic Evaluation of Pressure Ulcer Care: A Cost Minimization Analysis of Preventive Strategies. Nurs Econ. 27,390-400, 415(2009).

4. Ying L. et al. The prevalence, incidence, and associated factors of pressure injuries among immobile inpatients: A multicentre, cross-sectional, exploratory descriptive study in China. Int Wound J. 16, 459466(2019).

5. Schoonhoven, L., Defloor. T., Grypdonck M. H. F. Incidence of pressure ulcers due to surgery. J Clin Nurs. 11, 479-487(2002).

6. Wei, Y. S., Chen, J., Lu, Q., Wu, X. Y., Jiao, Y. J. Research progress on risk factors in assessing intraoperatively acquired pressure ulcers. Chinese Nursing Management.13,64-66(2013).

7. Galvin, P.A., Curley, M. A. Q. The Braden Q+ P: a pediatric perioperative pressure ulcer risk assessment and intervention tool. Aorn J. 96,261-270(2012).

8. Hayes, R.M. et al. Relationship between time in the operating room and incident pressure ulcers: a matched case-control study. Am J Med Qual. 30,591-597(2015).

9. Murry, C. E., Jennings, R. B., Reimer. K. A. Preconditioning with ischemia: a delay of lethal cell injury in ischemic myocardium. Circulation. 74,1124-1136(1986).

10. Thomas, S., Pulimood, A., Balasubramanian, K. A. Heat preconditioning prevents oxidative stressinduced damage in the intestine and lung following surgical manipulation. $\mathrm{Br} \mathrm{J}$ Surg. 90,473481(2003).

11. Mehta, S., Rolph, R., Cornelius, V., Harder, Y., Farhadi, J. Local heat preconditioning in skin sparing mastectomy: a pilot study. J Plast Reconstr Aesthet Surg. 66,1676-1682(2013).

12. Cro®S., Mehta, S., Farhadi, J., Coomber, B., Cornelius, V. Measuring skin necrosis in a randomised controlled feasibility trial of heat preconditioning on wound healing after reconstructive breast surgery: study protocol and statistical analysis plan for the PREHEAT trial. Pilot Feasibility Stud. 4,34(2018). 
13. Jing, H., Zou, G. M., Hao F. J., Wang, H. M., Wang, S.Z. Hsp27 reduces cold ischemia-reperfusion injury in heart transplantation through regulation of NF-KB and PUMA signaling. Int J Clin Exp Pathol. 11,281292(2018).

14. O'Neill, S., Hughes, J. Heat-shock protein-70 and regulatory T cell-mediated protection from ischemic injury. Kidney Int. 85,5-7(2014).

15. Ciocca, D. R., Steffi, O., Chamness, G. C., William L., M. C. Guire., Fuqua, S. A. W. Biological and clinical implications of heat shock protein 27000 (Hsp27): a review. J Natl Cancer Inst. 85,1558-1570(1993).

16. Boxman, I. L. A., Kempenaar, J., Haas, E. D., Ponec, M. Induction of HSP27 nuclear immunoreactivity during stress is modulated by vitamin C. Exp Dermatol. 11, 509-517(2002).

17. Hensbergen, P. J. et al. Proteomic profiling identifies an UV-induced activation of cofilin-1 and destrin in human epidermis. J Invest Dermatol. 124,818-824(2005).

18. Armour D. J., Preston-Hsu E., Tailor Y. Management of Pressure Ulcers and Pressure-Related Injury. Curr Phys Med Rehabil Rep. 7,170-177(2019).

19. Wilmink, G. J. et al. Molecular imaging-assisted optimization of hsp70 expression during laser-induced thermal preconditioning for wound repair enhancement. J Invest Dermatol. 129,205-216(2009).

20. Jonak, C., Klosner, G., Trautinger, F. Heat shock proteins in the skin[J]. Int J Cosmet Sci. 28,233241(2006).

21. Liu, Y. J. et al. The effects of HSP27 against UVB-induced photoaging in rat skin. Biochem Biophys Res Commun. 512,435-440(2019).

22. Muramatsu, T., Hatoko, M., Tada, H., Kobayashi, N., Shirai,T. Induction of the Low-Molecular-Weight Stress Protein HSP27 in Organ-Cultured Normal Human Skin. J Dermatol. 23,1-5(1996).

23. Singh, M. K., Sharma, B., Tiwari, P. K. The small heat shock protein Hsp27: present understanding and future prospects. J Therm Biol. 69:149-154(2017).

24. Mehta, S.et al. A randomised controlled feasibility trial to evaluate local heat preconditioning on wound healing after reconstructive breast surgery: the preHEAT trial. Pilot \& Feasibility Studies. 5:5(2019).

25. Scheel-Sailer, A. et al. Changes in skin-physiology after local heat application using two different methods in individuals with complete paraplegia: a feasibility and safety trial. Spinal Cord. 58,667674(2020).

26. Bauer, J., Phillips, L. G. Pressure Sores. Plast Reconstr Surg. 121(1 Suppl): 1-10(2008).

27. Thomas, D. R. Does pressure cause pressure ulcers? An inquiry into the etiology of pressure ulcers. $J$ Am Med Dir Assoc. 11,397-405(2010).

28. Chiu, J. H. et al. Preconditioned somatothermal stimulation on median nerve territory increases myocardial heat shock protein 70 and protects rat hearts against ischemia-reperfusion injury. $J$ Thorac Cardiovasc Surg. 125,678-685(2003).

29. Matsumoto, K., Honda, K., Kobayashi, N. Protective effect of heat preconditioning of rat liver graft resulting in improved transplant survival. Transplantation. 71,862-868(2001).

30. O'neill, S., Hughes, J. Heat-shock protein-70 and regulatory T cell-mediated protection from ischemic injury. Kidney Int. 85,5-7(2014). 
31. Marber, M. S. et al. Overexpression of the rat inducible 70-kD heat stress protein in a transgenic mouse increases the resistance of the heart to ischemic injury. J Clin Invest. 95,1446-1456(1995).

32. Yang, C. W. et al. Preconditioning with sodium arsenite inhibits apoptotic cell death in rat kidney with ischemia/reperfusion or cyclosporine-induced injuries. Exp Nephrol. 9, 284-294(2001).

33. Thomas, S., Prabhu, R., Pulimood, A., Balasubramanian, K. A. Heat preconditioning prevents enterocyte mitochondrial damage induced by surgical manipulation. J Surg Res. 108,138-147(2002).

34. Patel, H. H., Hsu, A., Gross, G. J. Cardioprotection is strain dependent in rat in response to whole body hyperthermia. Am J Physiol Heart Circ Physiol. 280, H1208-H1214(2001).

35 . Wilson, N. et al. Hyperthermia to normal human skin in vivo upregulates heat shock proteins $27,60,72 \mathrm{i}$ and 90. J Cutan Pathol. 27,176-182(2000).

36. Harder, Y. et al. Heat Shock Preconditioning Reduces Ischemic Tissue Necrosis by Heat Shock Protein (HSP)-32-Mediated Improvement of the Microcirculation Rather Than Induction of Ischemic Tolerance. Ann Surg. 242, 869-879(2005).

37. Boxman, I. L. A., Hensbergen $₫$ P. J., Schors $\bigotimes$ R. C. V. D., Bruynzeel『D. P., Ponec, M. Proteomic analysis of skin irritation reveals the induction of HSP27 by sodium lauryl sulphate in human skin. $\mathrm{Br} \mathrm{J}$ Dermatol. $146,777-785(2002)$.

38. Stadler, I., Zhang, R. Y., Oskoui, P., Whittaker, M. S., Lanzafame, R.J. Development of a simple, noninvasive, clinically relevant model of pressure ulcers in the mouse. J Invest Surg. 17,221-227(2004).

39. Zhang, B.G. Influences of Heat Shock Pretreatment on DNA Double-strand Breaks Damaged by High Temperature of V79 Cells. Cancer Research on Prevention and Treatment. 39,6-8(2012).

40. Chen, Y. J. et al. Macrophage-derived MMP-9 enhances the progression of atherosclerotic lesions and vascular calcification in transgenic rabbits. J Cell Mol Med. 24, 4261-4274(2020).

\section{Tables}

Table 1 Comparison between HPC group and control group [n(\%), mean $\pm S D]$

\begin{tabular}{|c|c|c|c|c|c|c|c|c|}
\hline Groups & $\begin{array}{l}\text { Age } \\
\text { (years) }\end{array}$ & Male & $\begin{array}{l}\text { Have } \\
\text { medical } \\
\text { history }\end{array}$ & $\begin{array}{l}\text { Supine } \\
\text { position }\end{array}$ & $\begin{array}{l}\text { Hemoglobin } \\
(\mathrm{g} / \mathrm{L})\end{array}$ & $\begin{array}{l}\text { Albumin } \\
\text { (g/L) }\end{array}$ & $\begin{array}{l}\text { Operative } \\
\text { Duration } \\
\text { (hours) }\end{array}$ & $\begin{array}{l}\text { BMI } \\
\left(\mathrm{kg} / \mathrm{m}^{2}\right)\end{array}$ \\
\hline \multirow{2}{*}{$\begin{array}{l}\text { Control } \\
(\mathrm{n}=59)\end{array}$} & \multirow{2}{*}{$\begin{array}{l}61.41 \pm \\
9.78\end{array}$} & 36 & 15 & 52 & $122.08 \pm$ & $40.03 \pm$ & $2.88 \pm$ & $18.94 \pm$ \\
\hline & & $\triangle 61.0 \% \square$ & $\triangle 25.4 \% \bigotimes$ & $\triangle 88.1 \% \bigotimes$ & 23.48 & 5.68 & 0.85 & 2.57 \\
\hline \multirow{2}{*}{$\begin{array}{l}\text { HPC } \\
\bigotimes n=59 \rrbracket\end{array}$} & \multirow{2}{*}{$\begin{array}{l}63.81 \pm \\
10.69\end{array}$} & 44 & 16 & 51 & $122.88 \pm$ & $39.71 \pm$ & $2.94 \pm$ & $21.44 \pm$ \\
\hline & & \74.6\%区 & $\triangle 27.1 \% \bigotimes$ & \86.4\%区 & 24.84 & 7.50 & 0.94 & 3.47 \\
\hline$t / c^{2}$ & $1.276^{\#}$ & $2.484^{\dagger}$ & $0.044^{\dagger}$ & $0.076^{\dagger}$ & $0.179^{\#}$ & $0.253^{\#}$ & $0.412^{\#}$ & $-0.465^{\#}$ \\
\hline$P$ & 0.205 & 0.115 & 0.834 & 0.782 & 0.858 & 0.801 & 0.681 & 0.643 \\
\hline
\end{tabular}


\# Represents $t$ value; ${ }^{\dagger}$ represents $c^{2}$ value; HPC, heat preconditioning;SD, standard deviation ; BMI, body mass index.

Table 2 Temperature comparison between HPC group and control group [n(\%), mean \pm SD]

\begin{tabular}{|llll|}
\hline Groups & $\begin{array}{l}\text { Before HPC } \\
\left({ }^{\circ} \mathrm{C}\right)\end{array}$ & $\begin{array}{l}\text { After HPC } \\
\left({ }^{\circ} \mathrm{C}\right)\end{array}$ & $\begin{array}{l}\text { Difference before } \\
\text { and after } \mathrm{HPC}\left({ }^{\circ} \mathrm{C}\right)\end{array}$ \\
\hline control $\triangle \mathrm{n}=59 \rrbracket$ & $33.12 \pm 1.39$ & $33.39 \pm 1.25$ & $0.27 \pm 1.75$ \\
\hline $\mathrm{HPC}(\mathrm{n}=59)$ & $33.17 \pm 1.48$ & $35.19 \pm 1.37$ & $2.16 \pm 1.42$ \\
\hline$t$ & 0.173 & 7.425 & 6.425 \\
$P$ & 0.863 & $<0.001$ & $<0.001$ \\
\hline
\end{tabular}

HPC, heat preconditioning; SD, standard deviation

Table 3 Outcomes comparison between HPC group and control group [n(\%)]

\begin{tabular}{|c|c|c|}
\hline Groups & Patients with PI & Healed \\
\hline Control $\otimes n=59 \rrbracket$ & $8 \rrbracket 13.60 \% \rrbracket$ & $2 \rrbracket 25.00 \% \rrbracket$ \\
\hline $\operatorname{HPC}(n=59)$ & $2 \bigotimes 3.40 \% \rrbracket$ & $2 \rrbracket 100.00 \% \rrbracket$ \\
\hline$c^{2}$ & 3.933 & 3.750 \\
\hline$P$ & 0.047 & 0.053 \\
\hline
\end{tabular}

HPC, heat preconditioning; PI, pressure injury

\section{Figures}




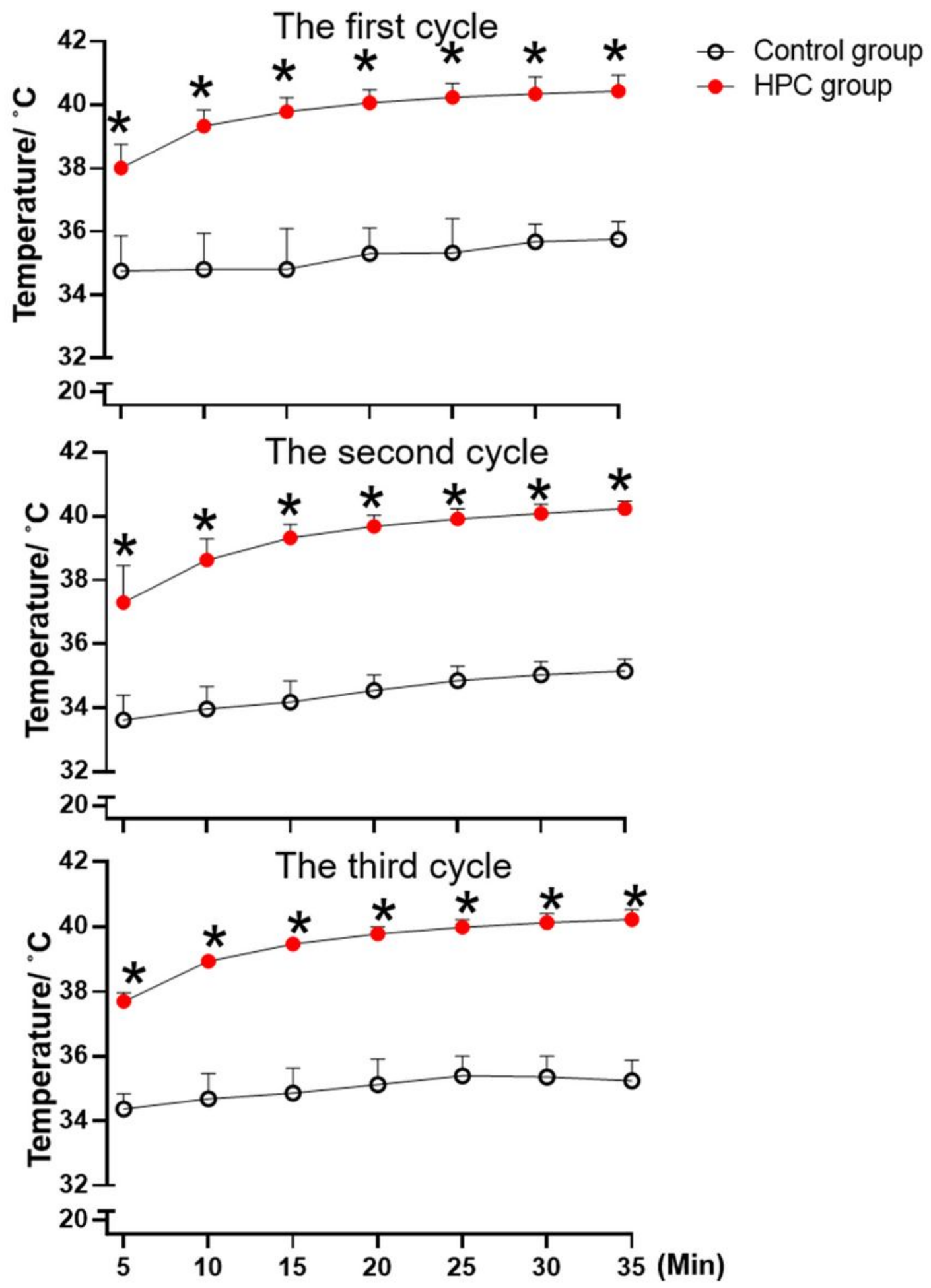

Figure 1

Changes of skin temperature in HPC group and control group in three cycles of heating preconditioning. $\mathrm{N}=7$, and data are expressed as the mean $\pm S D$. ${ }^{*} \mathrm{p} \otimes 0.05$, vs control group side. The independent-sample $T$ test was used. 


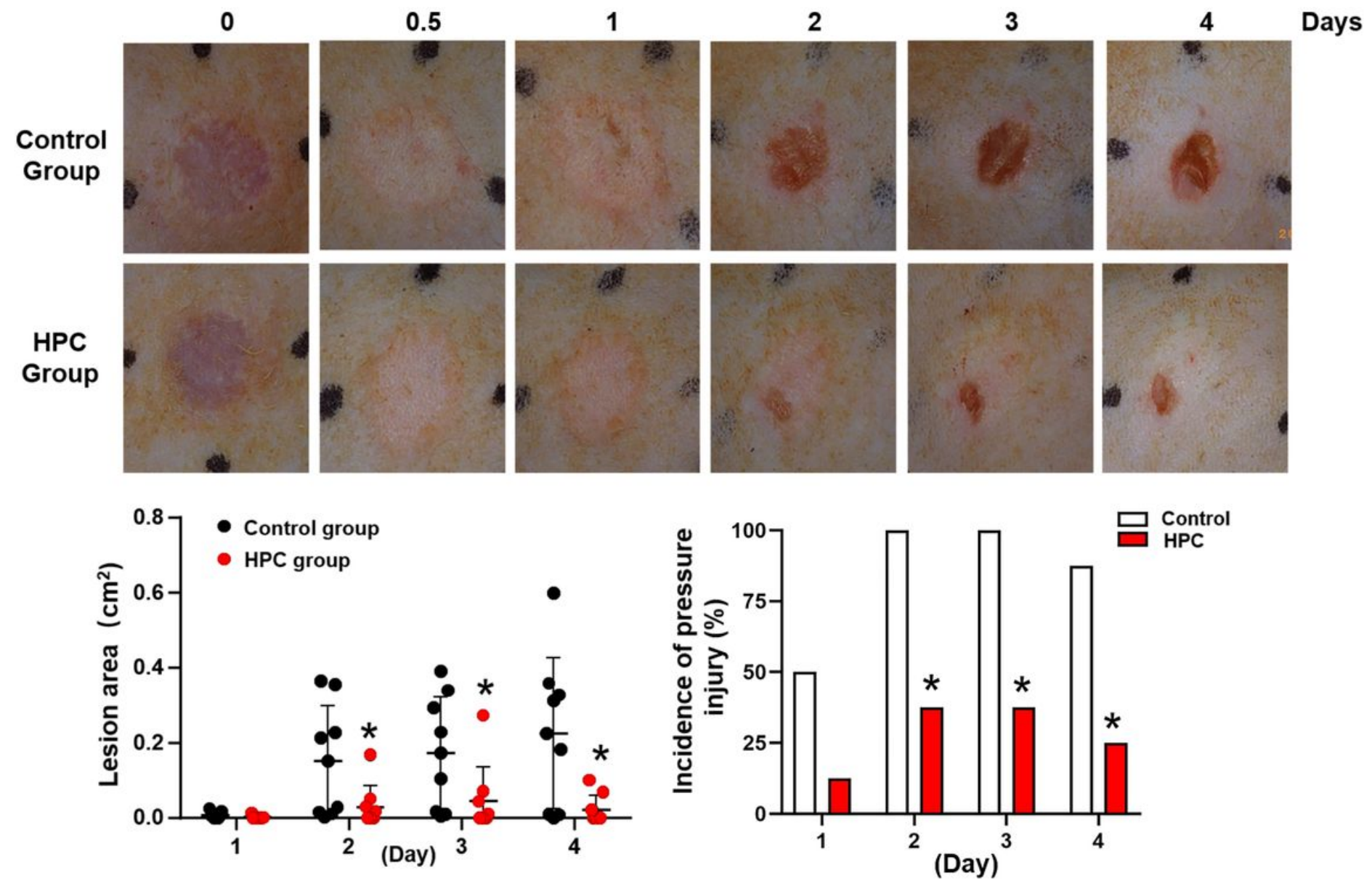

Figure 2

Representative pictures (top panel) and quantitation (bottom panel) of PI lesions at different time points. The effects of local HPC on the lesion area (bottom left) and incidence (bottom right) of PI in rats. Data are expressed as the mean $\pm S D$. $N=8$ for the control and HPC groups, and * $\otimes 0.05$, vs control group. The independent-sample $T$ test was used for lesion area and Fisher's Exact Test for incidence of pressure injury. 


\section{Non-injury area}

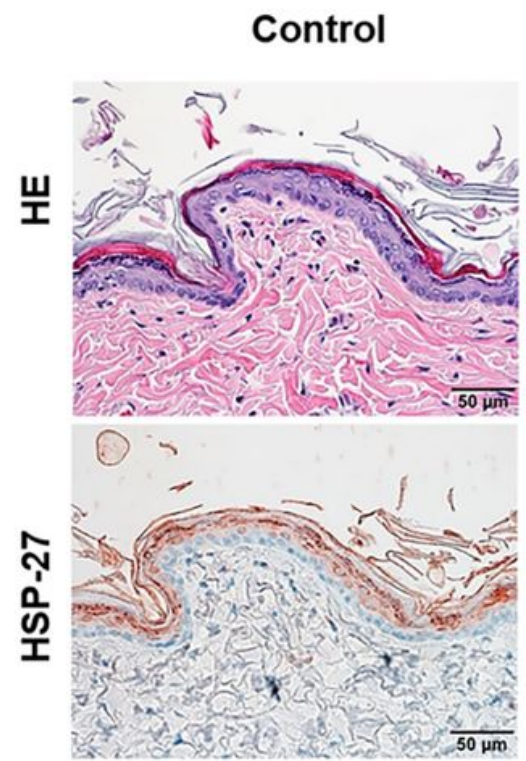

HPC
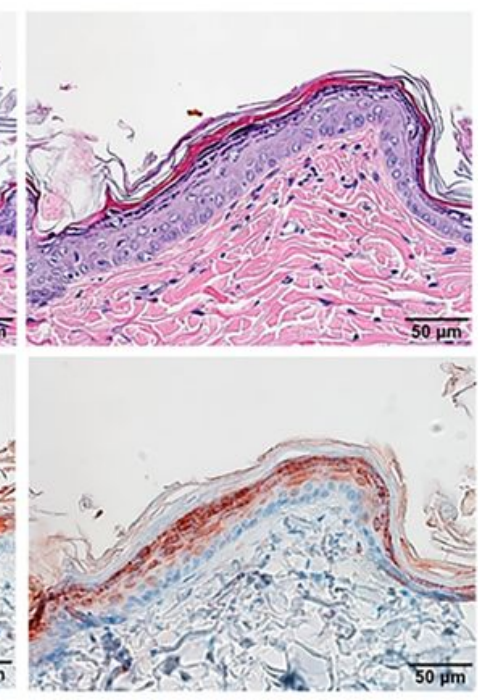

Injury area

Control

HPC
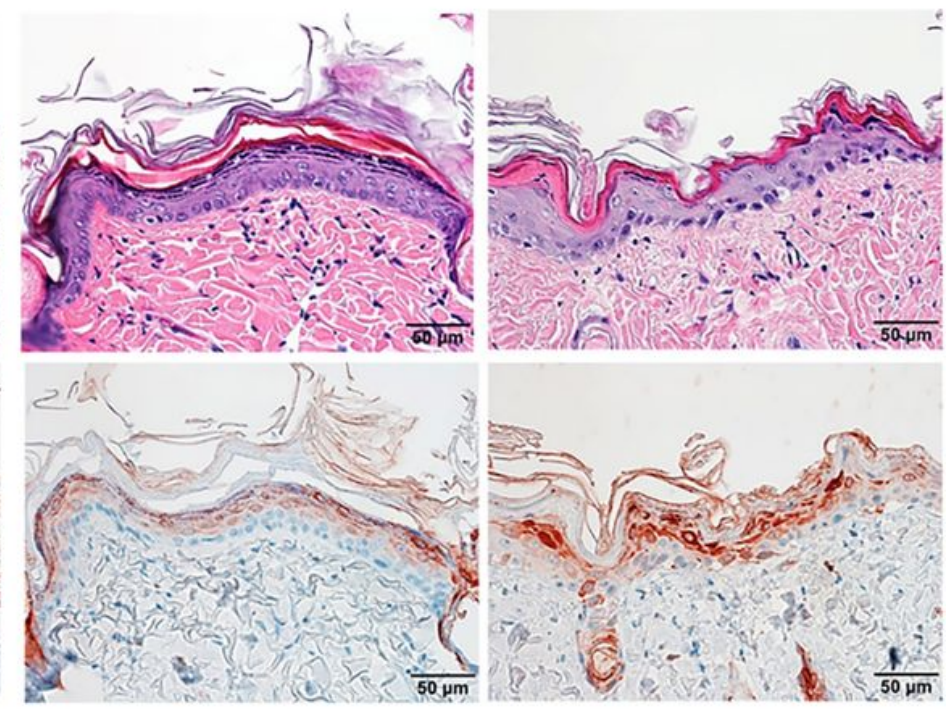

\section{Figure 3}

Representative images of skin in non-injury and injury area were shown here. Serial paraffin sections were stained with hematoxylin and eosin (HE, top panel) or immunohistochemical staining with HSP-27 (bottom panel). 

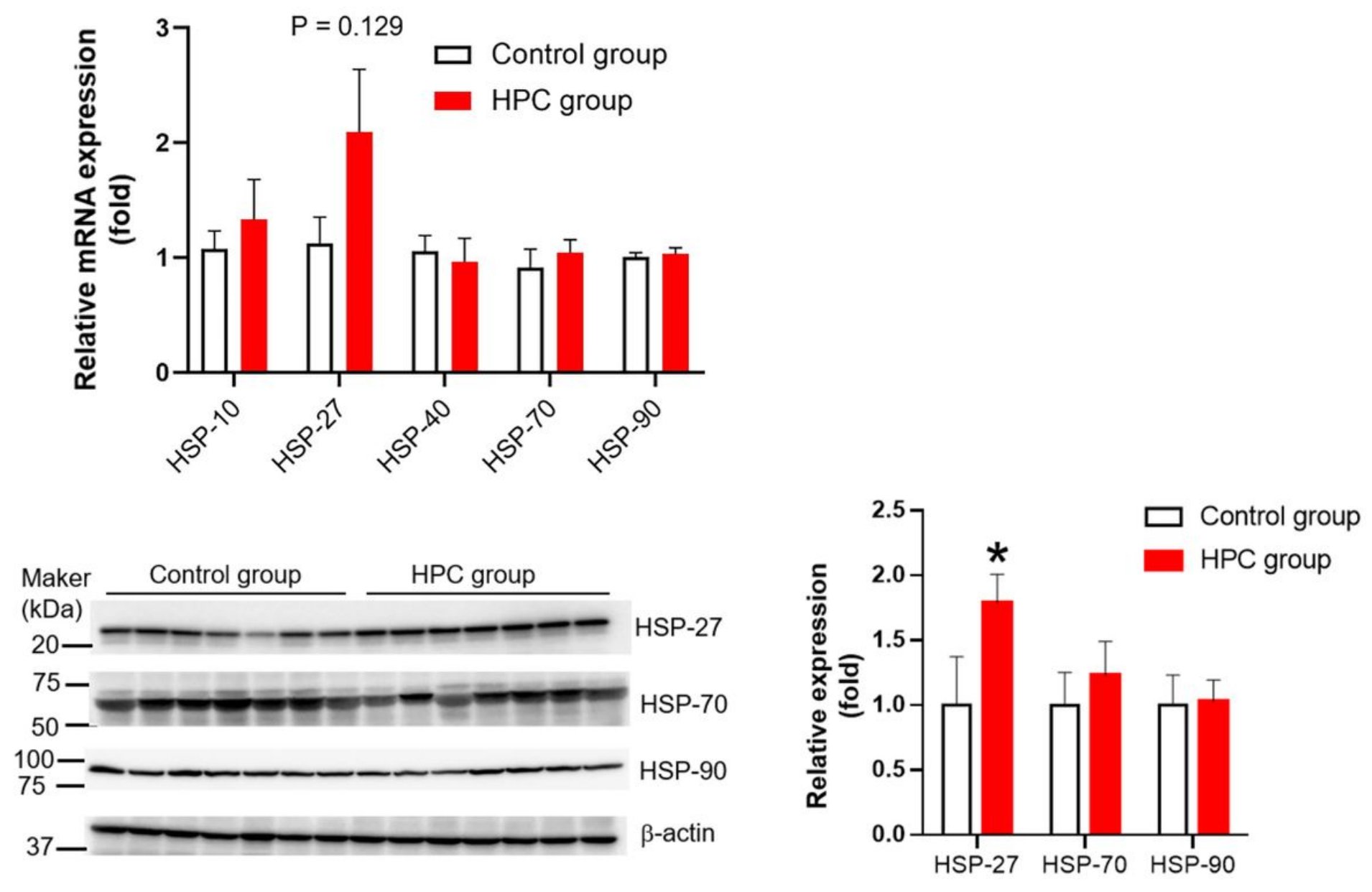

Figure 4

Expression of HSPs in PI lesion. mRNA expression of HSP-10, HSP-27, HSP-40, HSP-70 and HSP-90 were quantified using real-time RT-PCR (top panel). Data are expressed as the mean \pm SD. $n=7$ for each group. The effect of HPC on the induction of HSP27 protein in lesion area was analyzed by western blotting (bottom left) and quantified (bottom right). Data are expressed as the mean $\pm S D(n=7)$. * $p \otimes 0.05$, vs control group (independent-sample T test).

\section{Supplementary Files}

This is a list of supplementary files associated with this preprint. Click to download.

- SupplementaryFigureS1.tif

- SupplementaryFigureS2.tif 